\section{REFERENCES}

1 Warth A, Herpel E, Schmähl A, et al. Mediastinal angiomyolipomas in a male patient affected by tuberous sclerosis. Eur Respir J 2008; 31: 678-680.

2 Johnson SR, Whale CI, Hubbard RB, Lewis SA, Tattersfield AE. Survival and disease progression in UK patients with lymphangioleiomyomatosis. Thorax 2004; 59; 800-803.

3 Cornog JL Jr, Enterline HT. Lymphangiomyoma, a benign lesion of chyliferous lymphatics synonymous with lymphangiopericytoma. Cancer 1966; 19: 1909-1930.

4 Taylor JR, Ryu J, Colby TV, Raffin TA. Lymphangioleiomyomatosis: clinical course in 32 patients. N Engl J Med 1990; 323: 1254-1260.
5 Avila NA, Chen CC, Chu SC, et al. Pulmonary lymphangioleiomyomatosis: correlation of ventilation-perfusion scintigraphy, chest radiography, and CT with pulmonary function tests. Radiology 2000; 214: 441-446.

$6 \mathrm{Ng}$ CS, Wan S, Lee TW, Yim AP. Cystic lesions of the lung: a forgotten menace. Eur Respir J 2005; 26: 748-749.

7 Taveira-Da Silva AM, Stylianou MP, Hedin CJ, Hathaway O, Moss J. Decline in lung function in patients with lymphangioleiomyomatosis treated with or without progesterone. Chest 2004; 126: 1867-1874.

DOI: $10.1183 / 09031936.00050608$

\title{
Inhaled steroids and COPD
}

\section{To the Editors:}

We read with interest the paper by SuISSA et al. [1], in which he and his co-authors state that "the effectiveness of inhaled corticosteroids in treating chronic obstructive pulmonary disease remains doubtful." They review the reported efficacy of inhaled corticosteroids (ICS), noting a lack of pulmonary function improvement but reduction in exacerbation frequency, and a meta-analysis showing reduced mortality [2]. However, they conclude that these findings could be biased by methodological problems. One form of bias that they highlight is failure to follow patients after premature withdrawal from randomised clinical trials. SUISSA et al. [1] opine that the TORCH (Towards a Revolution in COPD Health) [3] and OPTIMAL [4] studies have provided important evolution in understanding these issues. While both studies used intent-to-treat analysis, at least for the primary end-points, TORCH showed a nonsignificant trend towards improved survival and OPTIMAL did not show significant differences in the proportion of patients experiencing at least one exacerbation over a 12-month period.

Perhaps a more important form of bias highlighted by SUISSA et al. [1] is the effect of withdrawal of previously prescribed medications on entry into a randomised clinical trial. In the TORCH study, $48 \%$ of patients recruited were previously taking ICS (although SuISSA et al. [1] report 51\%), whereas in OPTIMAL the proportion was 76\% (although SuISSA et al. [1] report $77 \%$ ). Because of the high frequency of patients already taking ICS on entry into these studies, they actually represent two comparisons: 1) continuation of ICS versus withdrawal of ICS, and 2) introduction of ICS versus no change in treatment. We agree that the results of this type of study are equally, if not more, likely to be influenced by the former comparison than the latter. Furthermore, as SUISSA et al. [1] point out, a difference due to the former comparison does not necessarily equate to efficacy in the conventional sense, as exemplified by the latter. In their analysis, SuISSA et al. [1] show a difference in exacerbation frequency due to withdrawal of ICS but no difference due to the introduction of ICS.

These issues are arguably just as pertinent in the interpretation of the INSPIRE (Investigating New Standards for Prophylaxis in
Reducing Exacerbations) study, a recently reported comparison between an ICS/long-acting $\beta$-agonist combination and tiotropium [5]. The INSPIRE study was remarkable for its run-in phase, during which patients were given prednisolone $30 \mathrm{mg}$ daily for 14 days to "standardize their COPD management before randomization" [5]. Rather than standardising chronic obstructive pulmonary disease management, this approach to study design surely represents a marked departure from recommended maintenance therapy for patients in clinically stable state and is, therefore, likely to amplify the phenomenon described by SuISSA et al. [1]. Furthermore, in the INSPIRE study the proportion of patients previously taking ICS was 50\%. We consider, therefore, that the INSPIRE study also falls into the category of studies described by SuISSA et al. [1] as potentially biased by methodological problems and its conclusions should be viewed with caution.

In their paper, SUISSA et al. [1] suggest that trials of tiotropium might be subject to the same type of bias due to withdrawal of previously prescribed anticholinergic inhaler therapy prior to randomisation, although, to date, there is no scientific evidence to support such an implication. Access to the database of tiotropium clinical trials should enable a meta-analysis of exacerbations in subgroups that had anticholinergic therapy withdrawn at entry, compared with those that were naïve to anticholinergic therapy. Should such an analysis of tiotropium studies show a similar benefit in either group, then it will be reasonable to conclude that the benefits of long-acting anticholinergic therapy are genuinely due to introduction of the therapy rather than its withdrawal. Implications with regard to the efficacy of tiotropium should be reserved until such an analysis is published.

\section{C.B. Cooper* and M. Decramer ${ }^{\#}$}

*David Geffen School of Medicine, University of California, Los Angeles, Los Angeles, CA, USA. "Katholieke Universiteit Leuven, Leuven, Belgium.

\section{STATEMENT OF INTEREST}

Statements of interest for all authors can be found at www.erj.ersjournals.com/misc/statements.shtml 


\section{REFERENCES}

1 Suissa S, Ernst P, Vandemheen KL, Aaron SD. Methodological issues in therapeutic trials of COPD. Eur Respir J 2008; 31: 927-933.

2 Sin DD, Wu L, Anderson JA, et al. Inhaled corticosteroids and mortality in chronic obstructive pulmonary disease. Thorax 2005; 60: 992-997.

3 Calverley PM, Anderson JA, Celli B, et al. Salmeterol and fluticasone propionate and survival in chronic obstructive pulmonary disease. $N$ Engl J Med 2007; 356: 775-789.
4 Aaron SD, Vandemheen KL, Fergusson D, et al. Tiotropium in combination with placebo, salmeterol, or fluticasonesalmeterol for treatment of chronic obstructive pulmonary disease: a randomized trial. Ann Intern Med 2007; 146: 545-555.

5 Wedzicha JA, Calverley PM, Seemungal TA, et al. The prevention of chronic obstructive pulmonary disease exacerbations by salmeterol/fluticasone propionate or tiotropium bromide. Am J Respir Crit Care Med 2008; 177: 19-26.

DOI: $10.1183 / 09031936.00030508$

\section{Prior tuberculin skin testing does not boost QuantiFERON-TB results in paediatric contacts}

\section{To the Editors:}

We read with interest the paper by LEYTEN et al. [1], which appeared in the June 2007 issue of the European Respiratory Journal (ERJ). LEYTEN et al. [1] showed that prior tuberculin skin tests (TST) do not induce false-positive QuantiFERON ${ }_{\mathbb{R}}-\mathrm{TB}$ Gold in-tube (QFT-GIT) assay results when evaluated in the days immediately following TST administration. In the same issue of the ERJ, NASEER et al. [2] reported the results of a study of 10 subjects without risk for tuberculosis infection, who were tested with QFT-GIT and the T-SPOT.TB assay (Oxford Immunotec, Abingdon, UK) before, $48 \mathrm{~h}$ and 6 weeks after TST administration. NASEER et al. [2] confirmed the results of LEYTEN et al. [1] in the first days following skin testing; however, after 6 weeks, three out of nine individuals turned from negative to positive with QFT-GIT, and none of the subjects turned from negative to positive with T-SPOT.TB. On this basis, NASEER et al. [2] state that there is evidence of boosting-specific interferon (IFN)- $\gamma$ responses 6 weeks after TST. According to these results, a high incidence of falsepositive results with QFT-GIT should be expected when the assay is performed weeks after the skin test. This issue is potentially very relevant in clinical practice, as current guidelines indicate that contacts recently exposed should be tested for latent infection at the time of diagnosis of the index case and, if negative, after 8-12 weeks [3]. We therefore retrospectively reviewed our series of paediatric contacts to identify an effect similar to the one reported by NASEER et al. [2].

A total of 70 children received both TST and QuantiFERON ${ }_{\mathbb{B}-}$ TB Gold (QFT-G); 51 (72.9\%) were QFT-G negative, nine (12.8\%) were QFT-G positive and 10 (14.3\%) were QFT-G indeterminate (low positive control). After 8-11 weeks, only one of the 51 initially QFT-G-negative contacts became QFT-G positive, and the mean values of IFN $-\gamma$ units produced after stimulation with early secretory antigenic target (ESAT)- 6 and culture filtrate protein (CFP)-10 did not differ before and after TST (ESAT-6 difference: $0.005 \mathrm{IU} \cdot \mathrm{mL}^{-1}, \quad \mathrm{p}=0.745 ; \mathrm{CFP}-10$ difference: $\left.0.030 \mathrm{IU} \cdot \mathrm{mL}^{-1}, \mathrm{p}=0.453\right)$. Of the 10 children with indeterminate QFT-G results, six tested indeterminate again, three became negative and one became positive. A total of 81 children were evaluated with TST and QFT-GIT. At the first visit, $63(77.8 \%)$ contacts were QFT-GIT negative, eight $(9.9 \%)$ were QFT-GIT positive and $10(12.3 \%)$ had an undetermined QFT-GIT. Of those initially negative children, only one became QFT-GIT positive after TST. The mean IFN- $\gamma$ antigen-specific production did not change 11 weeks after skin testing (ESAT-6/ CFP-10/TB7.7 difference $-0.030 \mathrm{IU} \cdot \mathrm{mL}^{-1}, \mathrm{p}=0.281$ ). Of the 10 subjects who were initially indeterminate with QFT-GIT, six remained indeterminate and four tested negative (table 1). Interestingly, as already reported in other studies [4, 5], we detected imperfect agreement between TST and QFT; in particular, the presence of TST-positive, QFT-negative children is similar to data recently published in an adult population [6]. Our findings show that among children at risk of acquiring latent tuberculosis infection after contact with an infectious index case, there is minimal or no effect on QFT several weeks after initial skin testing. Only two household contacts became QFT positive and both were heavily exposed to smear-positive cases; these QFT conversions could represent true recent infections, rather than sensitisation to purified protein derivative. Conversely, such a low rate of QFT conversions might be considered unexpected, and could be explained by the fact that at the time of index case diagnosis, most children already had prolonged contact and therefore may have undergone an antigen-specific T-cell response. At initial evaluation, $\sim 10 \%$ of contacts were QFT positive.

Our findings are consistent with those reported by LEYTEN et al. [1] but are in disagreement with those of NASEER et al. [2]; differences in size, demographic and clinical characteristics of study groups may account for this discrepancy. Our experience with IFN- $\gamma$ release assays for the diagnosis of latent tuberculosis supports the concept that prior administration of TST does not induce false-positive results of the blood tests. The lack of boosting effect of repeated TST on the enzymelinked immunospot-based blood assay has already been reported in 44 bacille Calmette-Guerin-unvaccinated TSTnegative adult and paediatric contacts repeatedly tested with the skin and blood assay over a 2 -yr period [7]. In that study, three conversions were observed with TST and none were observed with the blood assay. 